\title{
Simulating Minimally Invasive Neurosurgical Interventions Using an Active Manipulator
}

\author{
Arne Radetzky ${ }^{1}$, Michael Rudolph ${ }^{1}$, Werner Stefanon ${ }^{1}$, Stephen Starkie ${ }^{2}$, \\ Brian Davies ${ }^{2}$, and Ludwig M. Auer ${ }^{1}$ \\ ${ }^{1}$ Institute of Applied Sciences in Medicine, ISM-Austria, Jakob-Haringer Str. 3, A-5020 \\ Salzburg, Austria \\ \{A.Radetzky, Michael.Rudolph, Werner.Stefanon, Auer\}@ism- \\ austria.at \\ http://www.ism-austria.at \\ ${ }^{2}$ Department of Mechanical Engineering, Imperial College of Science, Technology and \\ Medicine, London, SW7 2BX, UK \\ \{s.starkie, b.davies\}@ic.ac.uk
}

\begin{abstract}
This application report describes the software system ROBO-SIM, which is a planning and simulation tool for minimally invasive neurosurgery. Using actual patients' datasets, ROBO-SIM consists of a planning unit and a simulator for microsurgical manipulations. The planning steps are 1. definition of the trepanation for entry into the intracranial space and virtual craniotomy, 2. the target point within the depth of the brain, 3. control of the surgical track, 4 . definition of go-areas for use with an intra-operative active manipulator. The simulator allows neurosurgeons to perform virtual surgical interventions using actual patient data and the same instruments as for the real operation.
\end{abstract}

\section{Introduction}

With the advent of computer-assisted methods in surgery, image-guided planning has become an increasingly accepted procedure in neurosurgery under the term of 'neuronavigation' [1-4]. As soon as robotic manipulators are considered to augment precision of microsurgical procedures [5-8], complex preoperative planning and simulation becomes mandatory and represent an important part of the total duration of a robotassisted operative procedure. An approach to combine a newly developed planning and simulation platform with the capability for robot-assisted neurosurgery is presented in the following. The integral setup called ROBO-SIM is designed for manipulator-assisted virtual procedures through a trepanation in the skull of $1-2 \mathrm{~cm}$ diameter and a miniaturized approach of a few millimeters diameter to target areas in the depth of the brain and its ventricular system. ROBO-SIM is part of the operating system ROBOSCOPE (project of the EU-Telematics program, No. 4.018), including a robot arm, NEUROBOT, on which the actual endoscope is mounted. The surgeon who plans and performs a virtual surgical procedure thus works with the real instruments also used during actual surgery, while looking at a virtual scenario of the operating field created by aid of a 3D-MRI-dataset of an actual patient. 


\section{NEUROBOT}

NEUROBOT (see Fig. 1), developed by Fokker control systems b.v., is used by the surgeon as an active manipulator with inbuilt robotic capabilities such as active constraints, within permitted regions, precise pattern control and the ability to automatically track features as they move and deform. NEUROBOT provides four degrees of freedom around a pivot point, which is the point of trepanation into the skull. Mounted to the robot is a force sensor with the ability to hold a standard neuro-endoscope. With the force sensor, every movement of the endoscope causes an active movement of the robot allowing a low-force input. In addition, rigid borders, e.g. pre-defined go-areas and no-go-areas, can be simulated using the active constraint mode. A foot switch controls the mode during operation. If it is activated, the robot can be moved; otherwise, the robot remains at its current position.
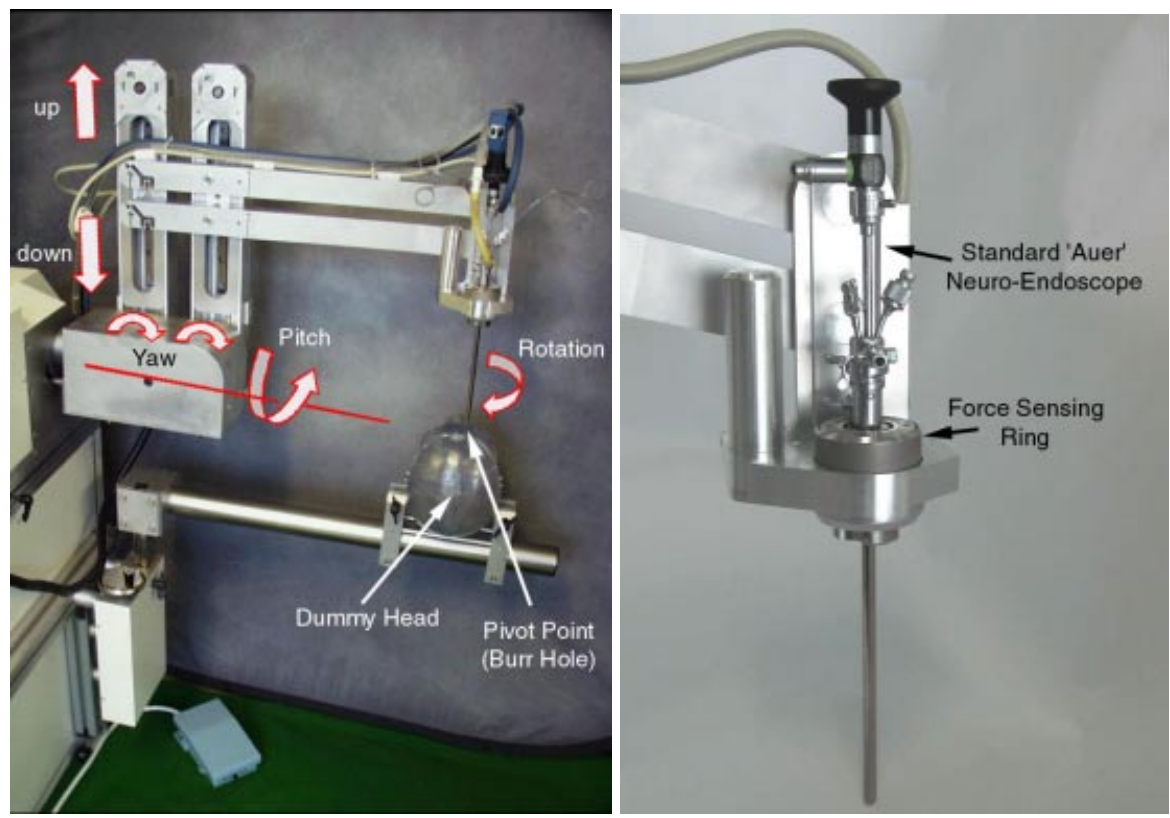

Fig. 1. Left: The active manipulator NEUROBOT provides four degrees of motion around a pivot point (burr hole). A dummy head can be clamped to the robot for simulated interventions. Right: A standard neuro-endoscope attached to a force sensing ring can be used to guide the robot. (Courtesy of Fokker Control Systems, B.V.)

NEUROBOT will be used either for real or simulated interventions. Thus, neurosurgical interventions can be trained using the same equipment as in real operations. For simulation, a dummy head can be clamped to the robot, which can be adjusted for different trepanation points. 


\section{ROBO-SIM}

To reduce the required amount of graphical power and nevertheless enable planning and simulation of neurosurgical procedures on actual patients' datasets, the use of a combination of volume- and surface-rendered data for visualization and simulation seems most practical. 3D-MRI datasets of the brains from actual patients are used to simulate views of the outer surface of the head as well as of inner surfaces, such as the ventricular system or cystic brain lesions, by aid of virtual endoscopy. For the simulations, 3D-MRI-datasets from a 1,5T Sigma echo-speed whole body scanner (GE Medical Systems, Milwaukee, USA) are used. The computer platform for the development and use of the system is an SGI Onyx2 Infinite Reality as well as SGI O2workstations and conventional PCs in several different program versions. The rendering engine is based on OpenGL and OpenGL Volumizer [9]. To speed-up the rendering process especially for virtual endoscopy, an extension of OpenGL Volumizer, called Flight-Volumizer, was developed [10]. Flight-Volumizer uses a field of view rendering facility, which allows only the data visible through the field of view of an endoscope to be rendered. In addition, simulations of deformations, fragmentations and coagulations are possible using surface rendering on segmented models of the ventricle.

ROBO-SIM consists of two main components: the planning tool and the simulation tool. Using the planning tool, all the steps necessary to preplan a minimally invasive neurosurgical intervention are included. These steps are 1. 'Define Entry- and Targetpoint' for planning of an approach and target point for the trepanation, 2. 'Check Surgical Track' for checking and changing the trajectory from entry to target point, 3 . 'Virtual Craniotomy' for simulating the trepanation and 4. 'Define Active Constraints' for defining go- and no-go-areas for robotic surgery.

\subsection{Define Entry and Target Point}

Usually the pre-operative planning begins with the definition of the point of entry into the skull for the trepanation (entry point) and the target point. Several pre-defined planning screens are included in ROBO-SIM consisting of a number of components. These components are two editors including axial, coronal and sagittal images of the patient for planning the entry and the target point, a virtual endoscopic view, a planning view, a main (global) view and several views with slices along the axis of the virtual endoscope. All screens are interactive and new screens can be defined by the neurosurgeon using the main components. Fig. 2 shows an example of such a planning screen. Moving the crosses for the entry or target point results in changing other slices and the virtual endoscopic view. The main or global view shows the position of the endoscope with respect to the patient's skull. 


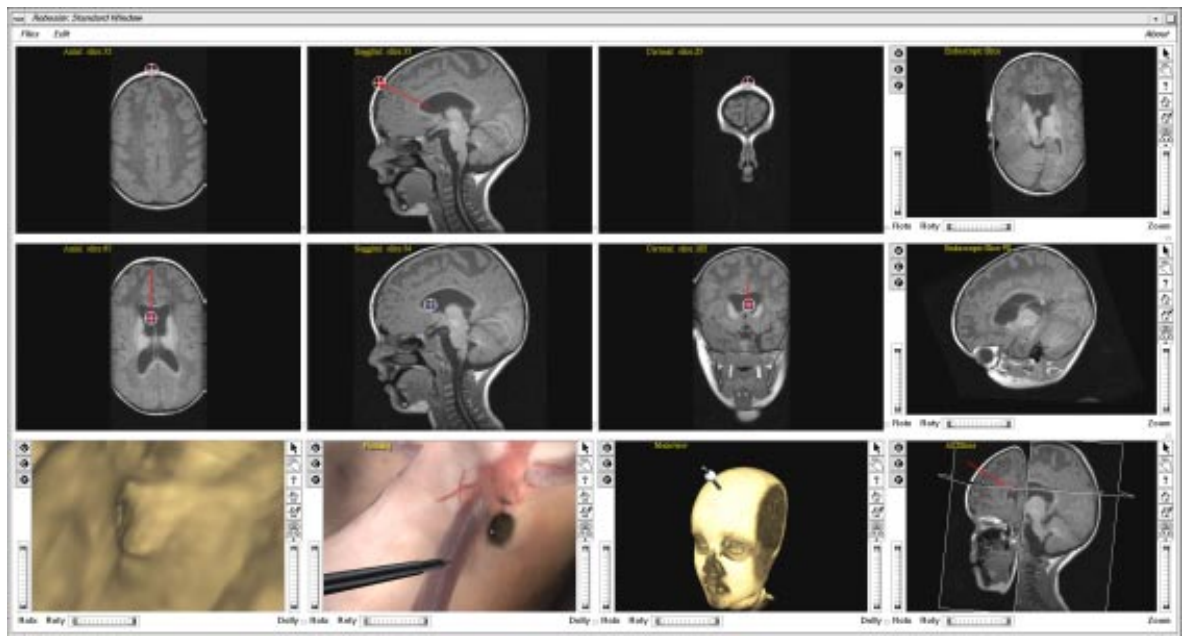

Fig. 2. An example screen of ROBO-SIM: The upper row of images allows the definition of the point of entry into the skull (trepanation). The middle row allows the definition of a requested target point. All images are primarily interactive; interaction may, however, be blocked. The image on the lower left shows a Flight-Volumizer virtual endoscopic view from a frontal approach into the left lateral ventricle. Lower middle images: view with a remote virtual endoscopic camera along the ventricular lumen shows the position of the virtual transendoscopic instrument and an outside view with a virtual endoscope in position for a left frontal approach. Right column: MR images along the endoscope and a virtual 3D view with the trajectory from entry to target point.

\subsection{Check Surgical Track and Virtual Craniotomy}

The next step is to check if the surgical track - i.e. the line from entry to target point avoids areas of the brain that should not be transgressed by the endoscope. The standard screen to check the surgical track consists of a main view and a virtual endoscopic view. The axial, sagittal and coronal images are also displayed, with marked red dots where the surgical track intersects the slices (see Fig. 3). These images are used to outline the track of the endoscope. The surgical track can be moved by dragging with the computer mouse.

After checking the surgical track, a virtual trepanation can be performed. The size, depth and position of the trepanation can be planned. The trepanation is directly and interactively visualized in the volume rendered outside view of the patient's skull (see Fig. 3). 


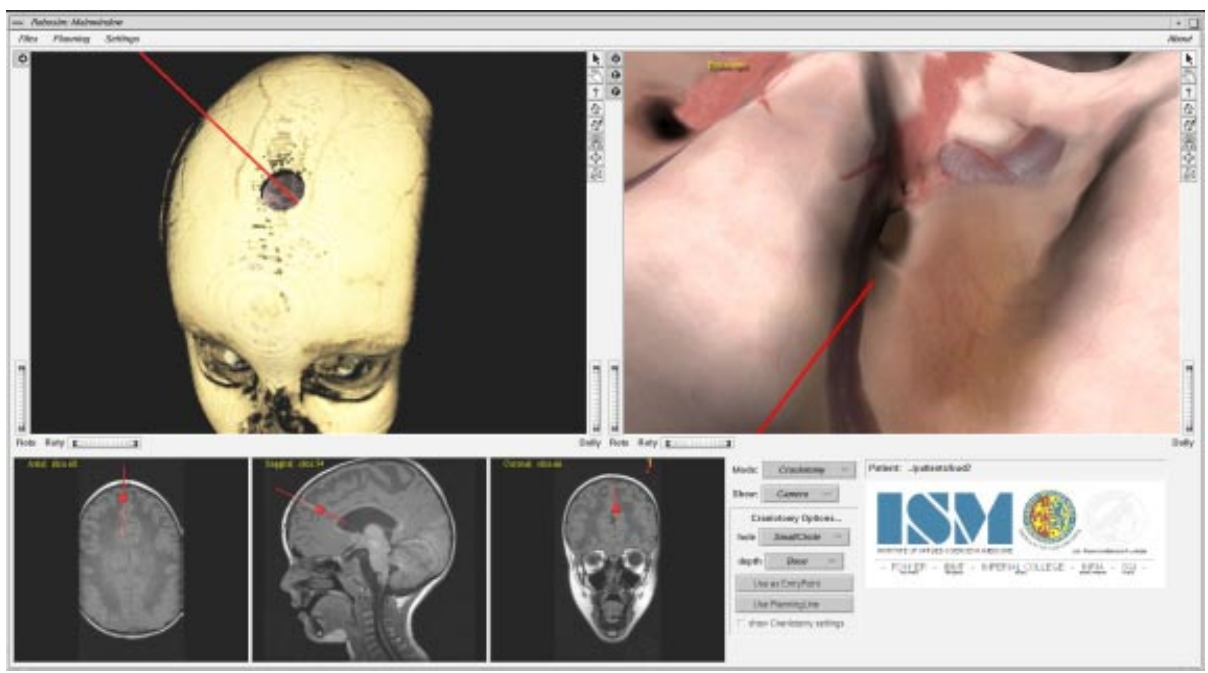

Fig. 3. The upper left image shows a volume-rendered outside view of the patient's dataset and the virtual trepanation at the entry point. The upper-right image shows the end of the surgical track. The surgical track can be moved with the mouse. The lower row shows axial, sagittal and coronal images of the patient's dataset overlaid with the surgical track and the point of intersection of the track and the image.

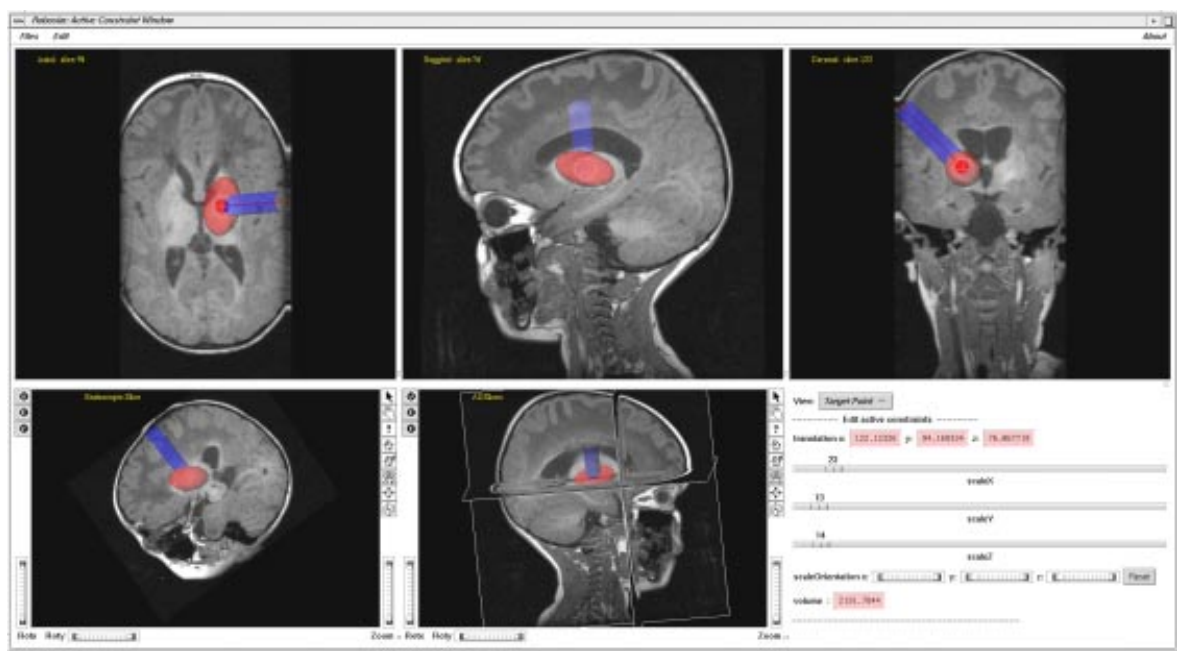

Fig. 4. The 'Active Constraint Screen' is used to define an arbitrary ellipsoid indicating the goarea for the active manipulator developed within the ROBOSCOPE project. The active manipulator restricts the surgeon performing a real operation from leaving this predefined area. The ellipsoid can be changed in size, orientation and position by the sliders on the lower right. A cylinder shows the direction from the entry to the target point. The radius of the cylinder indicates the radius of the trepanation. 


\subsection{Define Active Constraints}

The next step is to define the target area (see Fig. 4). This could be, for example, a brain tumor. The target area is used as active constraint for the active manipulator during an operation. To prevent the neurosurgeon from accidentally damaging healthy brain areas, physical resistance is provided to the surgeon by the motors embodied in the NEUROBOT. At present, arbitrary ellipsoids are allowed to define the target area.

\subsection{Simulation}

After finishing the planning steps, the simulation can be performed using the same active manipulator, which is used during a real operation. A volume rendered view of the ventricle can be used for real-time virtual endoscopy.

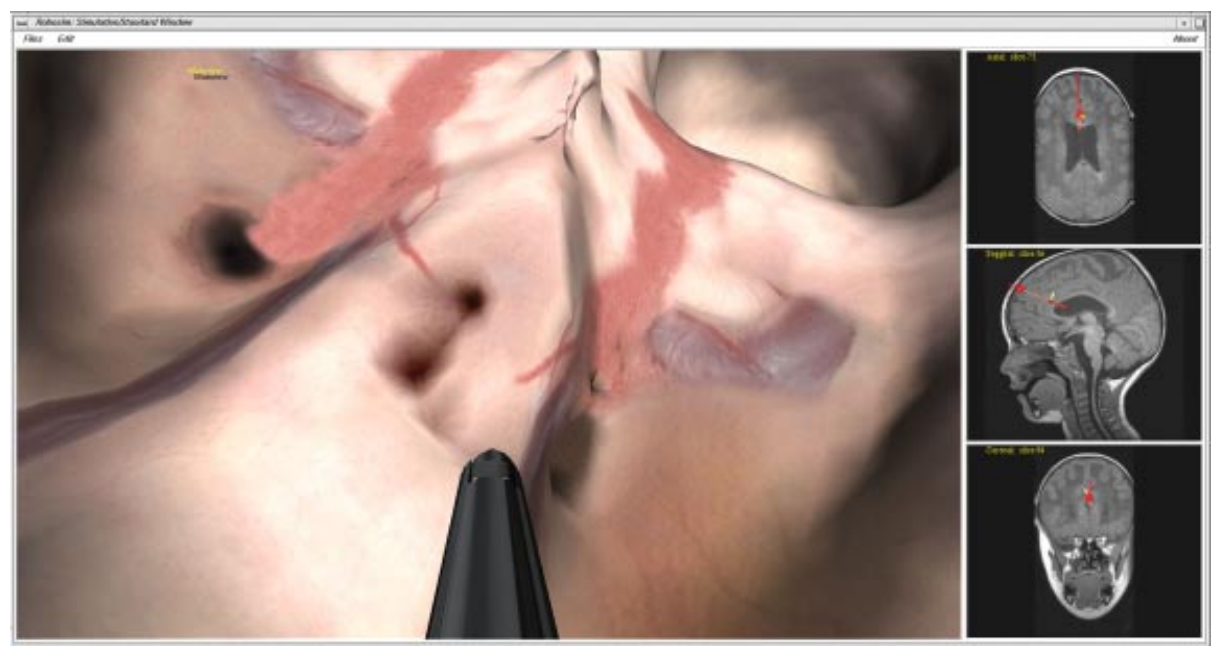

Fig. 5. Simulation screen of ROBO-SIM: Several tissue fragments were removed in center of the virtual endoscopic view by using a biopsy forceps. The right column shows the position of the endoscope in relation to the MR dataset and the surgical track.

In addition to simulation of virtual endoscopy, a full simulation tool based on deformable surface meshes was integrated in ROBO-SIM (see Fig. 5). The surface meshes are created by general object reconstruction based on simplex meshes [11]. For the physical-based simulation of these surface meshes, neuro-fuzzy systems [12] are used. These special neuro-fuzzy systems can emulate the real deformation even without a properly defined mesh structure [13]. Thus, volumetric meshes are not needed for realistic impressions of virtual deformations. In addition to the simulation of deformations, tools for coagulation and fragmentation of tissue are integrated in ROBO-SIM (see Fig. 6). Dynamic collision detection between the ventricle and the endoscope is performed using a cylinder-triangle collision test [14]. 


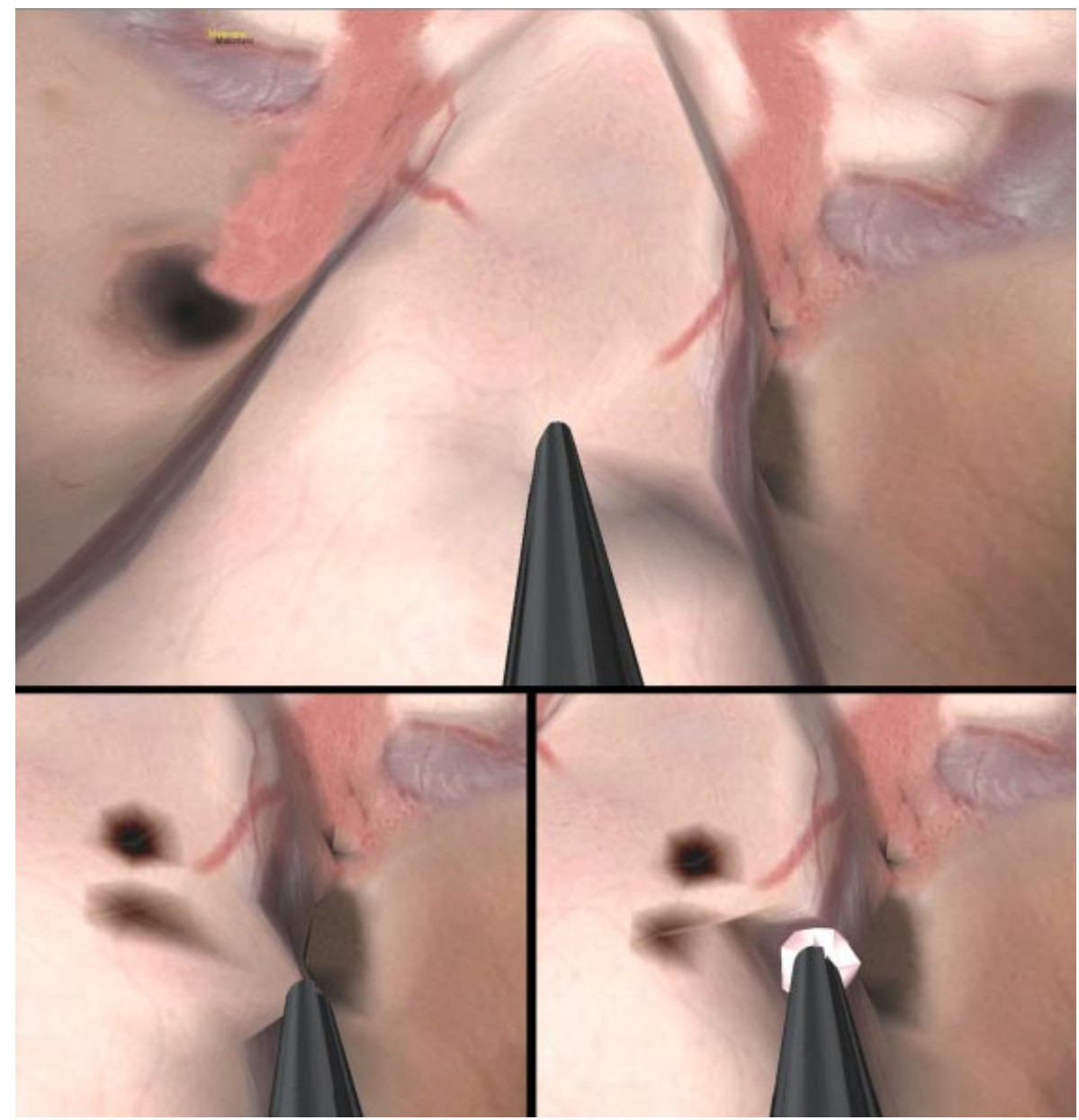

Fig. 6. Upper image: simulation of a deformation caused by a collision between the endoscopic instrument and the surface of the ventricle. Lower left image: parts of the tissue were already removed by the biopsy forceps (dark spots) and another part is gripped. Lower right image: The tissue is fragmented and the part that was ripped out, still remains in the biopsy forceps.

Using the real instruments mounted onto the robot arm, as well as the visual impression from the simultaneously moving virtual instruments and the virtual endoscopic anatomical landscape and its changes during manipulations, surgeons are able to simulate real procedures such as the removal of a tumor.

A first evaluation of ROBO-SIM on an actual patient dataset was already performed by an expert surgeon with very good results because of the high realism of the visualization and simulation. The only criticized points were the lack of blood flows and membranes. A more thoroughly test comparing the simulation results and the subjective opinions of a group of experienced neurosurgeons from all over Europe will follow. The neurosurgeons will fill in a questionary on subjective parameters concerning practicability, user friendliness and the quality of the simulation. 


\section{Conclusions}

The development of surgical simulators, comparable to flight simulators, has been initiated in a number of institutions (see, for example, [15-20]). Most of these simulators are working with simplified models of the human anatomy instead of using the anatomy of a real patient. ROBO-SIM is able to use directly the digital imaging datasets of the actual patient's neuro-anatomy. A simulation of minimally invasive neurosurgical procedures is considerably more complex than in most other areas, because the creation of the illusion of reality is required at a microsurgical level. Simulations, such as the real-time visualization of movements during manipulations, or the transfer of tactile sensations to the surgeon, or the visualization of the effect of robotic activities, provide a formidable challenge for high-end graphical computing and other disciplines.

To reduce the required amount of graphical power and nevertheless enable planning and simulation of neurosurgical procedures, the use of a combination of volume- and surface-rendered data for visualization and simulation seemed most practical in the hands of this group. 3D-MRI datasets of the brains from actual patients are used to simulate views of the outer surface of the head as well as of inner surfaces, such as the ventricular system or cystic brain lesions, by aid of virtual endoscopy. Although a direct manipulation of the MRI datsets is possible on high-end graphic systems [10], it has been proven that this approach is not useful simulating minimally invasive neurosurgical procedures. The MRI datasets show to less details and resolution for realistic impressions of deformations and fragmentation. Instead, the simulation works with surface models that represent a very exact model of the actual patient's ventricle.

At present ROBO-SIM is implemented on an Onyx2 IR (Silicon Graphics) with two MIPS1000 CPUs using the graphic library Open Inventor [21]. However, all of the algorithms, except the volume rendering, are already ported to a PC. In a first comparison, a two processor PC (Pentium III $800 \mathrm{MHz}$ ) with a fast 3D graphics board based on a NVIDIA Geforce2 chip was capable of a 50 percent higher simulation speed than the Onyx. The volume rendering is still a problem on the PC. However, the recently introduced VolumePro500 board allows orthographic real-time volume rendering on a PC [22]. Thus, porting ROBO-SIM to a PC seems a most promising task.

\section{Acknowledgements}

This project was partly supported by the Austrian Ministery of Research (grant to ISM) and by the European Commission, programme telematics (no. 4.018). The segmentations of the ventricles are done by algorithms provided by INRIA, Sophia Antipolis.

\section{References}

1. Auer LM, Auer D, Knoplioch JF. Virtual Endoscopy for Planning and Simulation of Minimally Invasive Neurosurgery. Lecture Notes in Computer Science 1205, SpringerVerlag 1997; 315 - 18 
2. Goradia TM, Kumar R, Taylor R, Auer LM. True Volumetric Stereotaxy for Intracerebral Hematomas. MMVR: Global Healthcare Grid: Transformation of Medicine through Communication. Amsterdam:IOS Press, 1999.

3. Auer DP, Auer LM. Virtual Endoscopy. A new Tool for Teaching and Training in Neuroimaging. Int.J. of Neuroradiol. 4, 1998, 3-14.

4. Auer LM, Auer, DP. Virtual Endoscopy for Planning and Simulation of Minimally Invasive Neurosurgery. Neurosurgery 43, 1998, 529-548.

5. Auer LM. Robots for Neurosurgery. In: Minimally Invasive Techniques for Neurosurgery. Current Status and Future Perspectives, eds.: Hellwig DH, Bauer BL, Springer Verlag 1998; 243-249

6. Benabid AL; Cinquin P; Lavalle S; Le-Bas JF; Demongeot J; De-Rougemont J. Computerdriven robot for stereotactic surgery connected to CT scan and magnetic resonance imaging. Technological design and preliminary results. APPL-NEUROPHYSIOL. 50/1-6 (153154) 1987

7. Davies BL, Ng WS, Hibberd RD, "Prostatic Resection; an example of safe robotic surgery." Robotica, Cambridge University Press, Vol 11, pp561-566, 1993.

8. Goradia, T.M., R Taylor, Auer LM. Robot-Assisted Minimally Invasive Neurosurgical Procedures: First Experimental Experience. Lecture Notes in Computer Science 1205, Springer 1997, 319-22

9. Grzeszczuk R, Henn C, Yagel R. Advanced Geometric Techniques for Ray Casting Volumes. In: Course Notes, 4, SIGGRAPH'98, 1998

10. Radetzky A, Schröcker F, Auer LM. Improvement of Surgical Simulation using Dynamic Volume Rendering. In: Medicine Meets Virtual Reality, Studies in Health Technology and Informatics, Amsterdam:IOS Press, 2000, 272-278

11. Delingette, H. General Object Reconstruction Based on Simplex Meshes. In: International Journal of Computer Vision, Boston, MA, 1999, 1-32.

12. Nauck D, Klawonn F, Kruse R. Foundations of Neuro-Fuzzy Systems, New York: John Wiley \& Sons Inc., 1997.

13. Radetzky A, Nürnberger A, Teistler M, Pretschner DP. Elastodynamic shape modeling in virtual medicine, In: International Conference on Shape Modeling and Applications, IEEE Computer Society Press, 1999, 172-178.

14. Karabassi E-A, Papaioannou G, Theoharis T und Boehm A. Intersection test for collision detection in particle systems. Journal of Graphics Tools 4(1), 1999, 25-37.

15. Jambon, A-C, Dubois P, Karpf S. A Low-Cost Training Simulator for Initial Formation in Gynecologic Laparoscopy. Troccaz J. Grimson E, eds. In: Proc. of CVRMed-MRCAS'97, Lecture Notes in Computer Science 1205, Berlin:Springer, 1997; 347-355.

16. Suzuki N, Hattori A, Kai S. Surgical Planning System for Soft Tissues Using Virtual Reality. In: Morgan KS, eds. Medicine Meets Virtual Reality: Global Healthcare Grid, Amsterdam: IOS Press, 1997; 39:159-163.

17. Bockholt U, Ecke U, Müller W, Voss G. Realtime Simulation of Tissue Deformation for the Nasal Endoscopy Simulator (NES). Westwood JD, Hoffman HM, Robb RA, Stredney D, eds. In: Medicine Meets Virtual Reality, Studies in Health Technology and Informatics 62, Amsterdam:IOS Press, 1999; 74-75.

18. Székely G, Bajka M, Bechbühler C, Dual J, Enzler R et al. Virtual Reality Based Surgery Simulation for Endoscopic Gynaecology. Westwood JD, Hoffman HM, Robb RA, Stredney D eds. In: Medicine Meets Virtual Reality, Studies in Health Technology and Informatics 62, Amsterdam:IOS Press, 1999; 351-357.

19. Bro-Nielson M, Tasto JL, Cunningham R, Merril GL. PreOp ${ }^{\mathrm{TM}}$ Endoscopic Simulator: A PC-Based Immersive Training System for Bronchoscopy. Westwood JD, Hoffman HM, Robb RA, Stredney D, eds. In: Medicine Meets Virtual Reality, Studies in Health Technology and Informatics 62, Amsterdam:IOS Press, 1999; 76-82. 
20. Ursino M, Tasto JL, Nguyen BH, Cunningham R, Merril GL. CathSim ${ }^{\mathrm{TM}}$ : An Intravascular Catheterization Simulator on a PC. Westwood JD, Hoffman HM, Robb RA, Stredney D eds. In: Medicine Meets Virtual Reality, Studies in Health Technology and Informatics 62, Amsterdam:IOS Press, 1999; 360-366.

21. Wernecke J. The Inventor Mentor. New York: Addison-Wesley, 1994.

22. Pfister H, Hardenberg J, Knittel J, Lauer H, Seiler L. The VolumePro Real-Time RayCasting System. In: Computer Graphics Proceedings, Annual Conference Series, ACM SIGGRAPH, 1999. 